\title{
Noise Exposure and Distortion Product Otoacoustic Emission Suprathreshold Amplitudes: A Genome-Wide Association Study
}

\author{
Joel Lavinsky ${ }^{a} \quad$ Guilherme Kasperbauer $^{a} \quad$ Ricardo F. Bento $^{b} \quad$ Aline Mendonça $^{a}$ \\ Juemei Wang ${ }^{c}$ Amanda L. Crow ${ }^{c}$ Hooman Allayee $^{c}$ Rick A. Friedman ${ }^{d}$ \\ aPostgraduate Program in Medicine: Surgical Sciences, Federal University of Rio Grande do Sul, Porto Alegre, Brazil; \\ ${ }^{b}$ Department of Otolaryngology, University of São Paulo, La Jolla, CA, USA; `Keck School of Medicine of USC, \\ Los Angeles, CA, USA; dUniversity of California, San Diego, CA, USA
}

\section{Keywords}

Animal models - Distortion product otoacoustic emission · Genome-wide association study · Noise-induced hearing loss · Noise

\begin{abstract}
Background: Although several candidate-gene association studies have been conducted to investigate noise-induced hearing loss (NIHL) in humans, most are underpowered, unreplicated, and account for only a fraction of the genetic risk. Mouse genome-wide association studies (GWASs) have revolutionized the field of genetics and have led to the discovery of hundreds of genes involved in complex traits. The hybrid mouse diversity panel (HMDP) is a collection of classic inbred and recombinant inbred strains whose genomes have been either genotyped at high resolution or sequenced. To further investigate the genetics of $\mathrm{NIHL}$, we report the first GWAS based on distortion product otoacoustic emission (DPOAE) measurements and the HMDP. Methods: A total of 102 strains $(n=635)$ from the HMDP were evaluated based on DPOAE suprathreshold amplitudes before and after noise exposure. DPOAE amplitude variation was set at 60 and $70 \mathrm{~dB}$ SPL of the primary tones for each frequency sepa-
\end{abstract}

karger@karger.com www.karger.com/aud

Karger $\frac{1}{\%}$
C 2021 The Author(s).

Published by S. Karger AG, Basel

This is an Open Access article licensed under the Creative Commons Attribution-NonCommercial-4.0 International License (CC BY-NC) (http://www.karger.com/Services/OpenAccessLicense), applicable to the online version of the article only. Usage and distribution for commercial purposes requires written permission. rately $(8,11.3,16,22.6$, and $32 \mathrm{kHz})$. These values provided an indirect assessment of outer hair cell integrity. Six-weekold mice were exposed for $2 \mathrm{~h}$ to $10 \mathrm{kHz}$ octave-band noise at $108 \mathrm{~dB}$ SPL. To perform local expression quantitative trait locus (eQTL) analysis, gene expression microarray profiles were generated using cochlear RNA from 64 hybrid mouse strains ( $n=3$ arrays per strain). Results: Several new loci were identified and positional candidate-genes associated with NIHL were prioritized, especially after noise exposure (1 locus at baseline and 5 loci after exposure). A total of 35 candidate genes in these 6 loci were identified with at least 1 probe whose expression was regulated by a significant ciseQTL in the cochlea. After careful analysis of the candidate genes based on cochlear gene expression, 2 candidate genes were prioritized: Eya1 (baseline) and Efr3a (post-exposure). Discussion and Conclusion: For the first time, an association analysis with correction for population structure was used to map several loci for hearing traits in inbred strains of mice based on DPOAE suprathreshold amplitudes before and after noise exposure. Our results identified a number of novel loci and candidate genes for susceptibility to NIHL, especially the Eya1 and Efr3a genes. Our findings validate the power of the HMDP for detecting NIHL susceptibility genes.

(c) 2021 The Author(s).

Published by S. Karger AG, Basel

Correspondence to:

Rick A. Friedman, rafriedman@ health.ucsd.edu 


\section{Introduction}

According to the Centers for Disease Control, up to $25 \%$ of the US adult population may develop noise-induced hearing loss (NIHL) [Kujawa and Liberman, 2009; Liberman, 2017]. In addition to human injury, NIHL generates a considerable economic impact. An estimated USD 1.2 billion is spent annually in the USA on disability compensation for NIHL [Yankaskas, 2013].

There is evidence for a genetic contribution to NIHL. A study evaluating the genetic component of NIHL in twins showed an approximately $36 \%$ contribution of inheritance to this condition [Heinonen-Guzejev et al., 2004]. Elucidating specific genetic effects is critical to understanding complex diseases such as NIHL. Although some studies of gene-environment interactions in human hearing loss have been conducted, only a few of them have been replicated to date [Lavinsky et al., 2015].

A major difficulty in human studies is the inability to control or quantify environmental exposures consistently, which results in a lack of well-characterized and wellmonitored populations for study. This is notably the case of NIHL, for which only a few ill-designed and poorly characterized studies have been published [Lavinsky et al., 2015]. For this reason, many researchers are turning to animal model organisms.

The study of hearing loss in mouse models appears to be a valid method since the mouse inner ear is structurally similar to the human inner ear, and their genetic material is homologous to a considerable extent. This can create an identical environment that avoids confounding factors and allows analysis through a genome-wide association study (GWAS) using inbred mouse lines from the hybrid mouse diversity panel (HMDP).

Studies evaluating NIHL in mice have characterized different susceptibility genes for NIHL using different methods [Sliwinska-Kowalska and Pawelczyk, 2013]. An example is the identification that some mouse strains (C57BL/6J) are more susceptible to noise than others [Erway et al., 1996]. Knockout mice tested by auditory brainstem response with targeted mutation of $\mathrm{SOD}^{-/-}$ [Ohlemiller et al., 1999], GPX1 ${ }^{-/-}$[Ohlemiller et al., 2000], and PMCA2 ${ }^{-/-}$[Kozel et al., 2002] were more sensitive to noise than their wild-type littermates. $\mathrm{CDH} 23^{+/-}$ mice were also characterized as more susceptible to noise [Holme and Steel, 2004], with their auditory function tested by measuring compound action potential thresholds.

Lavinsky et al. [2015], in a GWAS with correction for population structure, described for the first time the map- ping of several susceptibility loci for NIHL in inbred mouse strains using auditory brainstem response. The analysis revealed a genome-wide significant peak on chromosome 17 within a haplotype block containing NADPH oxidase-3, providing evidence to support its role in susceptibility to NIHL. In addition, frequency-specific genetic susceptibility to hearing loss after noise exposure was demonstrated within the mouse cochlea [Lavinsky et al., 2015].

Based on what has already been discovered, this study aimed to investigate other auditory phenotypes using a different method of auditory assessment, the distortion product otoacoustic emission (DPOAE) suprathreshold amplitude. This method indirectly assesses the outer hair cells, which are affected both before and after noise exposure.

\section{Materials and Methods}

\section{Hybrid Mouse Diversity Panel}

A detailed description of the HMDP (strain selection, statistical power, and mapping resolution) is provided by Bennett et al. [2010]. Four 4-week-old female mice of each HMDP strain were purchased from the Jackson Laboratory (Bar Harbor, ME). Only female mice were tested to avoid potential confounding effects of sex. The mice were allowed to acclimate to a common environment for 1 week (until 5 weeks of age). We used 5-week-old mice to minimize potential effects of age-related hearing loss that could contribute to our phenotype. All mice were fed a standard chow diet until sacrifice.

\section{Genotyping}

Classic inbred and recombinant inbred strains were previously genotyped by the Broad Institute (www.mousehapmap.org). Of the 140,000 single-nucleotide polymorphisms (SNPs) available, 108,064 were informative and used in the association analysis.

\section{Noise Exposure Protocol}

Using a method adapted from Kujawa and Liberman [2009], at 6 weeks of age, mice were exposed for $2 \mathrm{~h}$ to $10 \mathrm{kHz}$ octave-band noise at $108 \mathrm{~dB}$ SPL. The noise exposure protocol was previously described by White et al. [2009]. The cage was arranged in a soundproof chamber (MAC-1) created by Industrial Acoustics (IAC, Bronx, NY, USA). The sound chamber was lined with soundproofing acoustic foam. Noise recordings were performed with a speaker (Fostex FT17H Tweeter) built on the top of the sound chamber. Harmful noise was calibrated with a B\&K sound level meter, with a variation of $1.5 \mathrm{~dB}$ across the cage. The mice were placed in a circular exposure cage with 4 compartments, and for $2 \mathrm{~h}$, they were allowed to move around within the compartment. Testing involved the right ear only.

Determination of DPOAE Suprathreshold Amplitudes

DPOAEs were analyzed as input/output functions at $2 \mathrm{f} 1-\mathrm{f} 2$ (primary measure). The primary tones were set at a ratio of $\mathrm{f} 2 / \mathrm{f} 1$ 

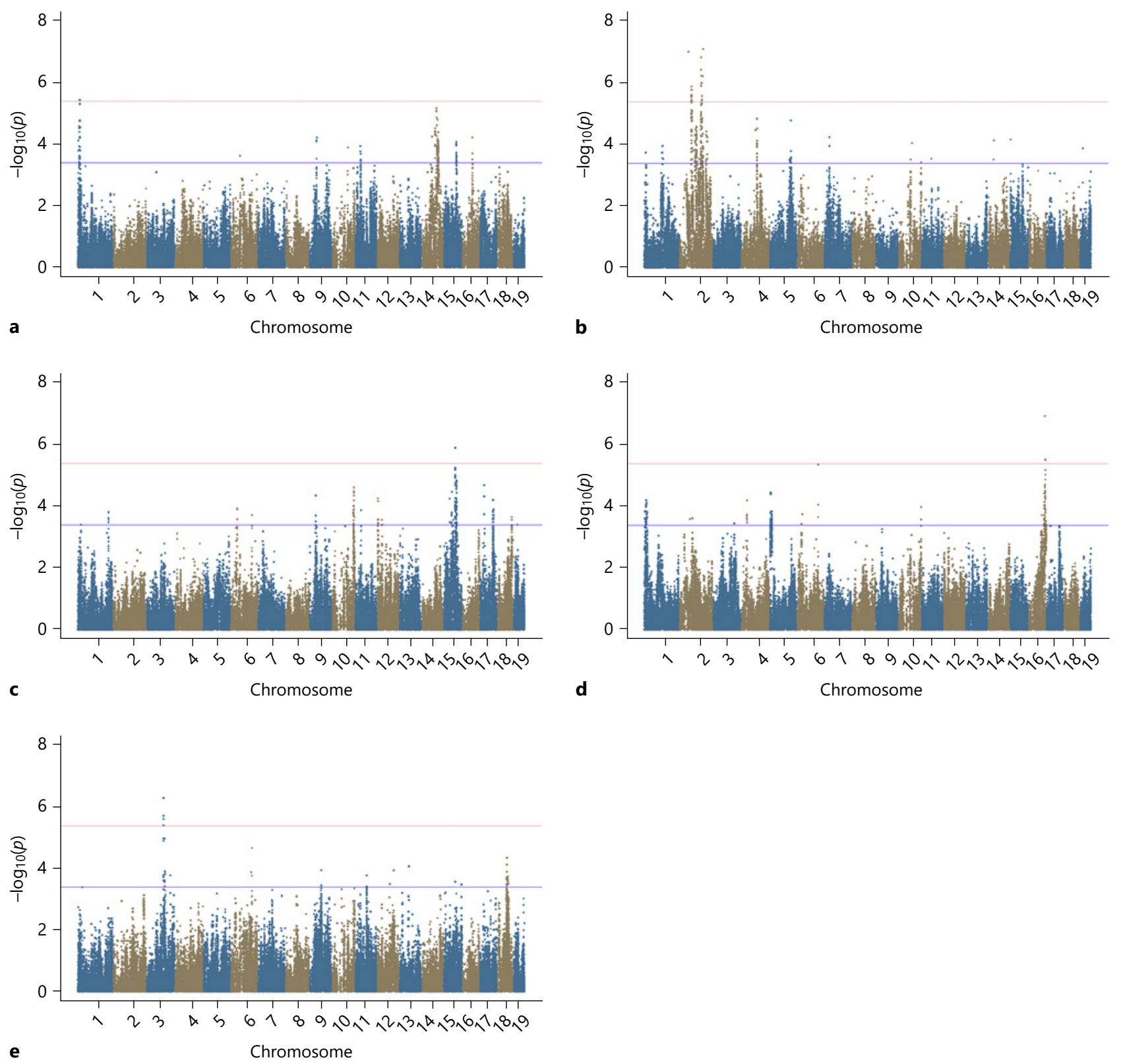

Fig. 1. Manhattan Plots: DPOAE GWAS. Association peaks $(-\log 10)$ of $p$ values $(-\log 10 \mathrm{p})$ for $32 \mathrm{kHz}-70 \mathrm{~dB}$ (a) before noise exposure and $8 \mathrm{kHz}-70 \mathrm{~dB}(\mathbf{b}), 11.3 \mathrm{kHz}-70 \mathrm{~dB}(\mathbf{c}), 16 \mathrm{kHz}-60 \mathrm{~dB}$ (d), and $32 \mathrm{kHz}-70 \mathrm{~dB}$ (e) after noise exposure (HMDP inbred strains). Analysis was performed using approximately 200,000 SNPs with minor allele frequency $>5 \%$. Each chromosome is plotted on the $x$-axis in alternating brown and blue colors. GWAS, genome-wide association study; DPOAE, distortion product otoacoustic emission; SNP, single-nucleotide polymorphism.

$=1.2$, with $\mathrm{f} 2$ ranging from 8 to $32 \mathrm{kHz}$ ( $\mathrm{f} 2$ level adjusted at $10 \mathrm{~dB}$ lower than $\mathrm{f} 1$ level) and $\mathrm{L} 2$ ranging from 60 to $70 \mathrm{~dB}$. The noise level was calculated as the average of 6 spectral points (above and below 2f1-f2). Both waveform and spectral mean DPOAEs were then extracted. The $\mathrm{L} 2$ level required to produce $0 \mathrm{~dB}$ SPL with signal-to-noise ratio was $3 \mathrm{~dB}$. DPOAE amplitude variation was set at 60 and $70 \mathrm{~dB}$ SPL of the primary tones for each frequency separately. These values provided an indirect assessment of outer hair cell integrity. 
Table 1. Association peaks of candidate genes within cis-regulated eQTLs in the cochlea

\begin{tabular}{|c|c|c|c|c|c|}
\hline Gene & RefSeq & Chr & txStart, bp ${ }^{a}$ & txEnd, bp ${ }^{b}$ & Local eQTL $p^{\mathrm{C}}$ \\
\hline$X k r 9$ & ILMN_3159558 & 1 & 13668771 & 13701723 & $1.79 \mathrm{E}-01$ \\
\hline Eyal & ILMN_2638487 & 1 & 14168954 & 14310200 & $6.26 \mathrm{E}-03$ \\
\hline$M s c$ & ILMN_2769777 & 1 & 14753346 & 14755966 & $2.50 \mathrm{E}-04$ \\
\hline Mapkbp1 & ILMN_2873793 & 2 & 119972699 & 120027408 & $3.77 \mathrm{E}-02$ \\
\hline Pla2g4d & ILMN_3156124 & 2 & 120265595 & 120289197 & $4.79 \mathrm{E}-02$ \\
\hline Ltk & ILMN_1219860 & 2 & 119751320 & 119760431 & $6.37 \mathrm{E}-02$ \\
\hline Rpap1 & ILMN_2602151 & 2 & 119763304 & 119787537 & $1.65 \mathrm{E}-02$ \\
\hline Pla2g4f & ILMN_2727722 & 2 & 120299957 & 120314165 & $1.03 \mathrm{E}-03$ \\
\hline Vps39 & ILMN_3129752 & 2 & 120316461 & 120353137 & $1.52 \mathrm{E}-02$ \\
\hline Tmem $87 a$ & ILMN_2969172 & 2 & 120355312 & 120404113 & $1.57 \mathrm{E}-02$ \\
\hline Ganc & ILMN_2791355 & 2 & 120403896 & 120461700 & $1.85 \mathrm{E}-01$ \\
\hline$Z f p 106$ & ILMN_2482427 & 2 & 120506820 & 120563843 & $2.30 \mathrm{E}-01$ \\
\hline $\operatorname{Tgm} 5$ & ILMN_2690147 & 2 & 121046111 & 121085841 & $3.14 \mathrm{E}-01$ \\
\hline Ccndbp1 & ILMN_1240178 & 2 & 121008403 & 121016904 & $3.18 \mathrm{E}-01$ \\
\hline Adal & ILMN_1242772 & 2 & 121140428 & 121156680 & $3.35 \mathrm{E}-01$ \\
\hline $\operatorname{Trp} 53 b p 1$ & ILMN_1243753 & 2 & 121193281 & 121271407 & $3.80 \mathrm{E}-02$ \\
\hline Tubgcp4 & ILMN_1257622 & 2 & 121170654 & 121198770 & $3.82 \mathrm{E}-01$ \\
\hline Adcy8 & ILMN_2607127 & 15 & 64699042 & 64922296 & $3.79 \mathrm{E}-01$ \\
\hline Olig1 & ILMN_2760105 & 16 & 91269769 & 91271939 & $2.14 \mathrm{E}-06$ \\
\hline Ifnar2 & ILMN_2598703 & 16 & 91485238 & 91507441 & $6.66 \mathrm{E}-07$ \\
\hline Gpr89 & ILMN_2953098 & 3 & 96868281 & 96905346 & $3.07 \mathrm{E}-02$ \\
\hline$C d 160$ & ILMN_2707181 & 3 & 96798763 & 96829351 & $1.49 \mathrm{E}-02$ \\
\hline Fmo5 & ILMN_1230890 & 3 & 97628804 & 97655282 & $3.52 \mathrm{E}-01$ \\
\hline Chd1l & ILMN_2986172 & 3 & 97560742 & 97610203 & $1.57 \mathrm{E}-02$ \\
\hline Pde4dip & ILMN_3064283 & 3 & 97689824 & 97888707 & $4.00 \mathrm{E}-01$ \\
\hline Ankrd34a & ILMN_3020599 & 3 & 96596636 & 96599775 & $6.43 \mathrm{E}-02$ \\
\hline$R b m 8 a$ & ILMN_1252850 & 3 & 96629928 & 96633779 & $6.80 \mathrm{E}-02$ \\
\hline Pex11b & ILMN_1258372 & 3 & 96635376 & 96653101 & $1.64 \mathrm{E}-01$ \\
\hline$S v 2 a$ & ILMN_1213495 & 3 & 96181151 & 96195521 & $3.01 \mathrm{E}-01$ \\
\hline Mtmr11 & ILMN_2676662 & 3 & 96162004 & 96171718 & $3.77 \mathrm{E}-01$ \\
\hline Hist $2 h 2 a c$ & ILMN_1218380 & 3 & 96220361 & 96220880 & $4.10 \mathrm{E}-01$ \\
\hline Hist $2 h 2 b e$ & ILMN_1231066 & 3 & 96221121 & 96223738 & $9.24 \mathrm{E}-03$ \\
\hline $\operatorname{Prpf3}$ & ILMN_2941430 & 3 & 95830124 & 95855885 & $5.17 \mathrm{E}-02$ \\
\hline ВС028528 & ILMN_2656422 & 3 & 95883954 & 95892005 & $2.21 \mathrm{E}-01$ \\
\hline Aph1a & ILMN_1232821 & 3 & 95893969 & 95898288 & $3.09 \mathrm{E}-02$ \\
\hline
\end{tabular}

eQTL, expression quantitative trait locus. ${ }^{a}$ txStart, location of transcription (NCBI, Build 37 genome assembly) start. ${ }^{\mathrm{b}}$ txEnd, location of transcription (NCBI, Build 37 genome assembly) end. ${ }^{\mathrm{c}}$ Statistically significant $p$ value $\leq \mathrm{E}-06$ (Bonferroni corrected for the number of probes tested).

\section{Results}

Genome-Wide Association Analysis of NIHL Reveals Frequency-Specific Genetic Susceptibility

The efficient mixed-model association (EMMA) algorithm was applied to each phenotype separately to identify genetic associations for the 6 tone-burst stimuli [Kang et al., 2008]. Adjusted association $p$ values were calculated for the 108,064 SNPs with minor allele frequency $>5 \%$ $(p<0.05$ genome-wide equivalent for genome-wide association using EMMA in the HMDP is $p=4.1 \times 10^{-6}$, - $\log 10 p=5.39)$. At this threshold, genome-wide significant associations were identified on chromosome 1 at baseline and on chromosomes 2, 3, 15, and 16 after noise exposure.

\section{GWAS and DPOAE Suprathreshold Amplitudes at Each Frequency Tested}

An association analysis was performed for each phenotype separately in order to identify genetic associations. A GWAS was performed both at baseline (Fig. 1a) and after noise exposure (Fig. 1b-e) to identify NIHLassociated loci at the various frequencies tested. 


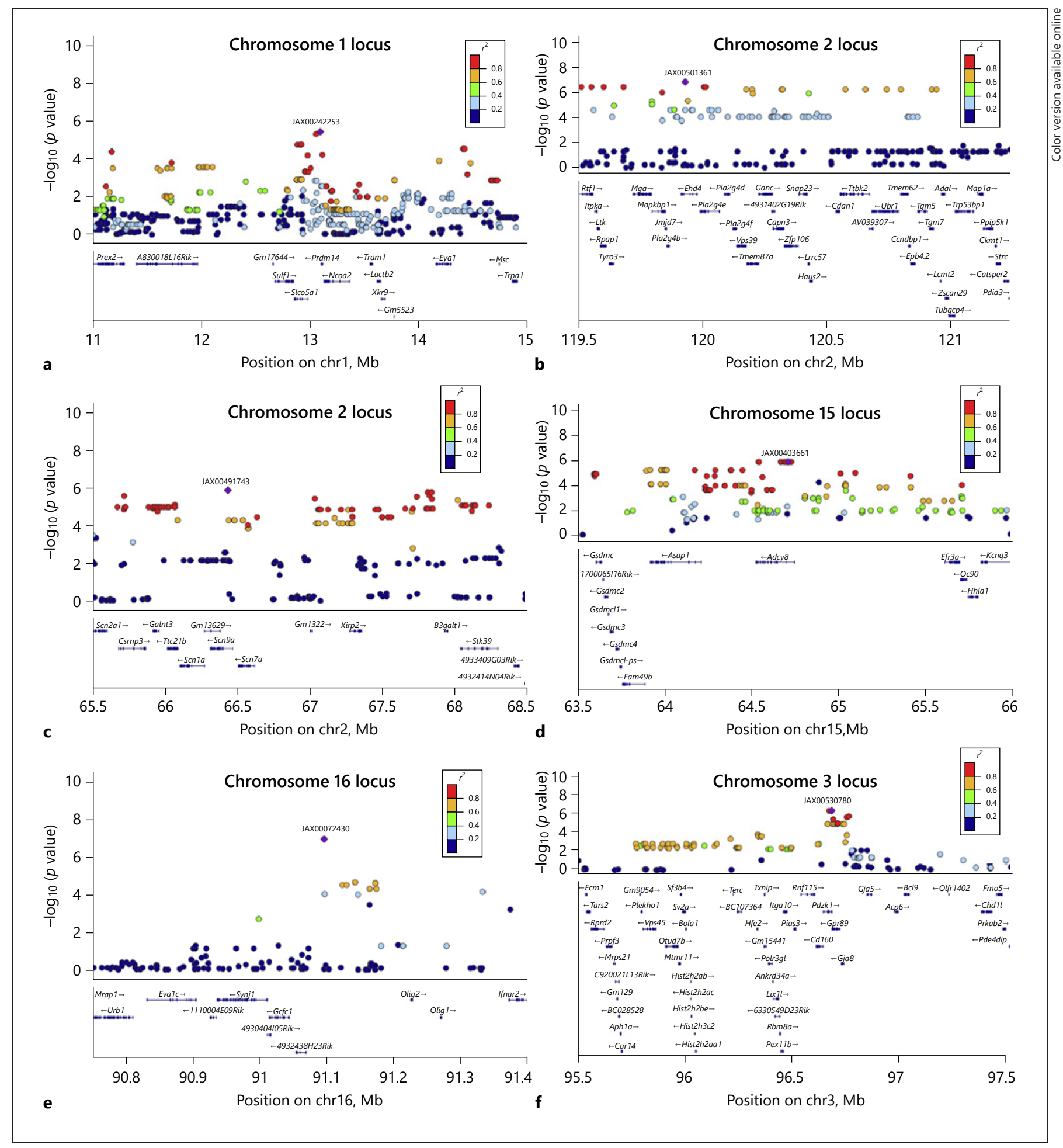

Fig. 2. Association peak centered on the lead SNP for $32 \mathrm{kHz}-70 \mathrm{~dB}$ (a) before noise exposure and $8 \mathrm{kHz}-70$ $\mathrm{dB}(\mathbf{b}, \mathbf{c}), 11.3 \mathrm{kHz}-70 \mathrm{~dB}(\mathbf{d}), 16 \mathrm{kHz}-60 \mathrm{~dB}(\mathbf{e})$, and $23 \mathrm{kHz}-70 \mathrm{~dB}(\mathbf{f})$ after noise exposure (HMDP inbred strains). The blue diamond represents the most significant SNP. Other SNPs are colored based on their LD, as follows: red SNPs in LD at $r^{2}>0.8$, orange SNPs in LD at $r^{2}>0.6$, and green SNPs in LD at $r^{2}>0.4$. The positions of all RefSeq genes are plotted using genomic sites (NCBI Build 37). HMDP, hybrid mouse diversity panel; LD, linkage disequilibrium; SNP, single-nucleotide polymorphism. 
Table 2. Biological processes and phenotypes associated with cis-eQTL candidate genes

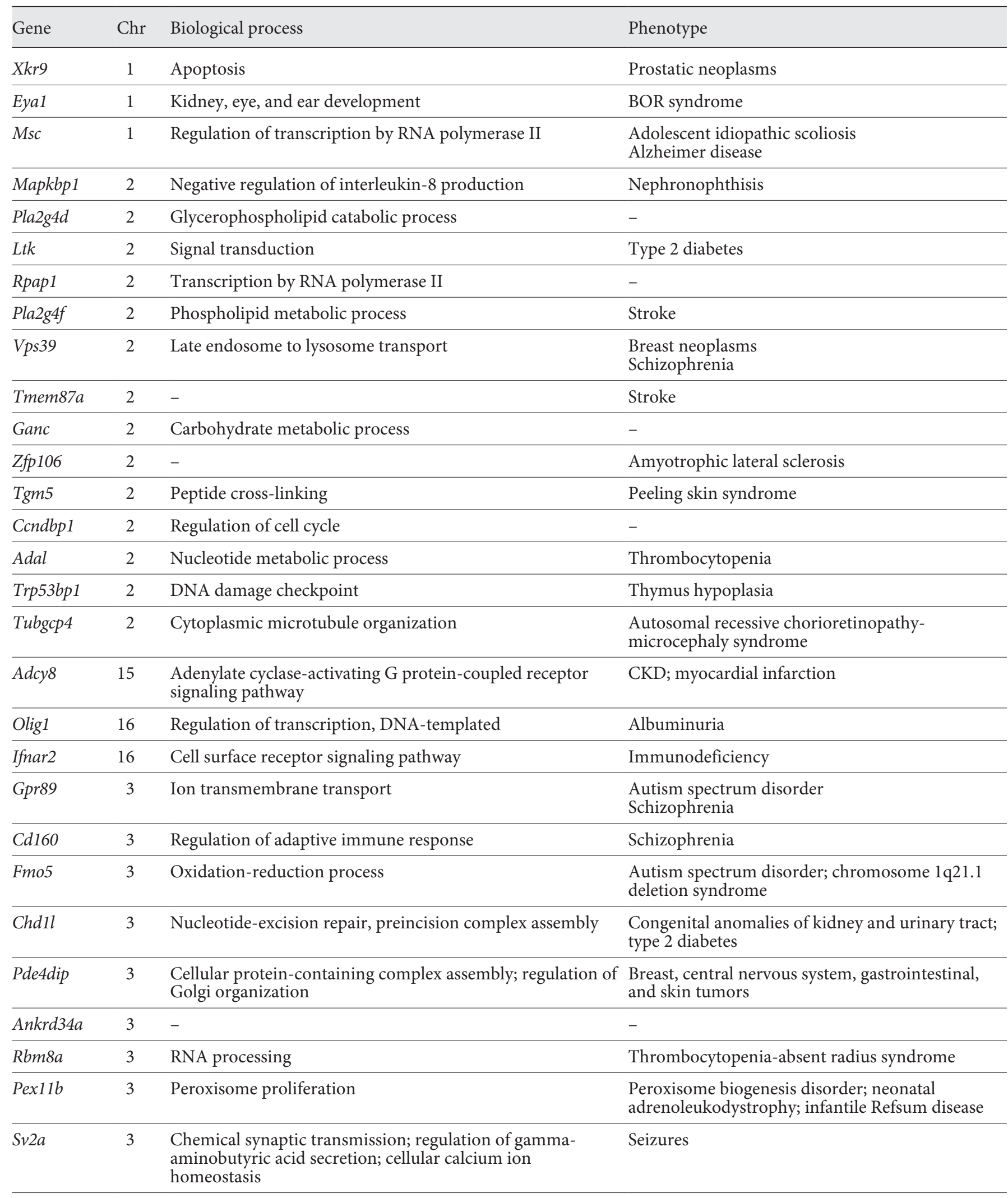


Table 2 (continued)

\begin{tabular}{llll}
\hline Gene & Chr & Biological process & Phenotype \\
\hline Mtmr11 & 3 & Phosphatidylinositol dephosphorylation & Adolescent idiopathic scoliosis \\
\hline Hist2h2ac & 3 & DNA binding & - \\
\hline Hist2h2be & 3 & $\begin{array}{l}\text { Antibacterial humoral response; negative regulation of } \\
\text { tumor necrosis factor-mediated signaling pathway }\end{array}$ & - \\
\hline Prpf3 & 3 & RNA binding & Retinitis pigmentosa \\
\hline BC028528 & 3 & - & - \\
\hline Aph1a & 3 & Notch receptor processing; beta-amyloid precursor protein & Bipolar disorder; schizophrenia \\
\hline
\end{tabular}

Retrieved from ensembl.org. Copyright 2020. eQTL, expression quantitative trait locus; BOR, branchio-oto-renal.

Association Analysis and Regional Plots

At baseline, a DPOAE association exceeding the genome-wide significance threshold was identified on chromosome 1 (Fig. 2a). After exposure, the associations were statistically significant on chromosome 2 (Fig. 2b, c), chromosome 15 (Fig. 2d), chromosome 16 (Fig. 2e), and chromosome 3 (Fig. 2f).

\section{Prioritizing Our Candidate Genes}

Our cochlear expression data allowed us to analyze all 144 candidate genes in each association interval. Genes were identified within each of the intervals regulated by a local expression quantitative trait locus (eQTL). In order to perform eQTL analysis, gene expression microarray profiles were generated using cochlear RNA from 64 hybrid mouse strains ( $n=3$ arrays per strain). Subsequently, EMMA was used to perform an association analysis between all SNPs and array probes mapping within each region. After excluding the probes that overlapped SNPs present among the classic inbred strains used in the HMDP, as described in the methods, 18,138 genes were represented by at least 1 probe.

Loci in which peak SNPs mapped within $2 \mathrm{Mb}$ of the gene whose expression was regulated were considered "local" or cis-acting eQTLs, while SNPs mapped elsewhere were considered "distal" and presumably transacting eQTLs. The significant $p$ value cutoff $\left(p=1 \times 10^{-6}\right)$ was calculated for local and distal associations. These genes were prioritized based on whether they were regulated by a local eQTL.

A total of 35 genes in the 6 identified loci had at least 1 probe whose expression was regulated by a significant cis-eQTL in the cochlea (Table 1). The functions of candidate genes and associated diseases are shown in Table 2.

\section{Discussion}

This study evaluated pre- and post-noise exposure auditory variation in mice by means of genome-wide association analysis using the HMDP and DPOAE suprathreshold amplitudes. The HMDP has been previously used successfully in the genomic mapping of blood cell traits in mice [Davis et al., 2013], in genomic association and systems genetics in mice by identifying $A s x l 2$ as a regulator of bone mineral density and osteoclastogenesis [Farber et al., 2011], in the genetic regulation of atherosclerotic plaque size and morphology in the innominate artery of hyperlipidemic mice [Bennett et al., 2009], in the identification of gene networks associated with conditional fear in mice using a systems genetics approach [Park et al., 2011], in the genetic control of obesity and gut microbiota composition in response to high-fat, high-sucrose diet in mice [Parks et al., 2013], in the identification of NADPH oxidase- 3 as a critical gene for susceptibility to NIHL [Lavinsky et al., 2015], and in diabetes [Parks et al., 2013] and heart failure [Rau et al., 2015]. In many of these studies, including the present GWAS, the use of modified mouse models allowed the genes in the identified loci to be validated as the causal genes, and several of them matched loci identified in human GWASs.

The present GWAS generated significant associations in 1 region at baseline and in 5 separate regions after exposure to noise, resulting in 144 candidate genes of which 35 in these 6 loci were identified with at least 1 probe whose expression was regulated by a significant cis-eQTL in the cochlea. After careful analysis of the candidate genes based on cochlear gene expression and after a review of the current literature, 2 main candidate genes 
were prioritized: Eya1 (baseline) and Efr3a (post-exposure).

The Eyal gene, which was present with a significant association peak at baseline at $32 \mathrm{kHz}-70 \mathrm{~dB}$ intensity, is a gene-encoding protein that acts on other genes, thus being considered a transcription factor. It is interesting to note that the so-far identified functions of Eyal correlate with certain forms of genetic hearing loss in humans and normal cochlear development [Xu et al., 2017]. The Eya1 protein interacts with several other proteins by activating and inactivating genes that are important for normal development. Key roles include the development of the second branchial arch, which gives rise to the tissues of the neck, eyes, ears, and kidneys [Kochhar et al., 2007].

The Eyal gene has grown in prominence with the discovery of its expression in mammalian cochlear stem cells. A population of Eyal-expressing progenitor cells capable of differentiating into hair cells, supporting cells, and ganglion cells after hair cell ablation up to 1 week of age was identified in mice. The identification of cochlear regeneration after birth in mammals and the characterization of this cell population (Eya1-positive) provided a new cellular source with potential for cochlear repair and regeneration [Xu et al., 2017].

Furthermore, Eyal gene mutations are the most common cause of the branchio-oto-renal syndrome. More than 80 pathogenic mutations were identified in Eyal in different populations [Zhang et al., 2004]. Branchio-otorenal syndrome is a rare autosomal dominant disorder characterized by sensorineural, mixed, or conductive hearing loss, branchial fistulas or cysts, preauricular pits, ear malformations, and renal anomalies.

Another important correlation was the confirmation that the inactivation of Eyal and Sixl in mice results in a significant alteration in ear development owing to an early interruption of development at the otocyst stage [Zheng et al., 2003]. Another robust evidence was the discovery of the involvement of Eya1/Six1 in hair cell development and their coexpression in mouse cochlear explants. Expression of these genes was sufficient to induce hair cell fate in the nonsensory epithelium expressing low-level Sox 2 by activating not only Atoh1-dependent but also Atoh1-independent pathways, and both pathways induce Pou $4 \mathrm{f} 3$ to promote the differentiation of hair cells [Ahmed, 2012].

$E f r 3 a$ is our main candidate gene, which was found at the frequency of $11.3 \mathrm{kHz}$ at $70 \mathrm{~dB}$ after noise exposure. It encodes a plasma membrane protein whose insufficiency has been initially reported to increase the survival of hippocampal neurons in adult mice. This is the first correlation of the gene with NIHL, which has been further investigated in age-related hearing loss and ototoxicity. These findings are in agreement with previous studies investigating Efr $3 a$ expression in the auditory neural pathway, which is upregulated soon after hair cell destruction. The impact of Efr $3 a$ insufficiency has also been evaluated by the functional and morphological comparison of the cochlea between Efr3a-deficient mice and their wild-type littermates. The present study suggests that Efr3a deficiency is associated with less severe hearing loss after ototoxic exposure and confirms greater morphological damage to cochlear spiral ganglion neurons. These beneficial effects of $E f r 3 a$ deficiency can be attributed to increased expression of some neurotrophic factors and their receptors, thus suppressing neurodegeneration $[\mathrm{Hu}$ et al., 2017].

Our finding is also consistent with another recent finding from research into age-related hearing loss that Efr $3 a$ deficiency results in better hearing function in senescent mice (at 10 and 12 months of age). It was demonstrated that Efr $3 a$-deficient senescent mice had less severe degeneration of cochlear spiral ganglion neurons in the basal turn; when degeneration occurred, it occurred later $[\mathrm{Hu}$ et al., 2016].

Moreover, ototoxic injury in mice showed to rapidly induce hair cell loss, resulting in progressive degeneration of the spiral ganglion 5 days after drug administration. The number of spiral ganglion cells began to decrease on day 15. Efr3a expression was notably increased in the spiral ganglion on day 5 , decreasing to near normal levels by day 10. This suggests that the change in Efr3a expression in the spiral ganglion coincides with the time of spiral ganglion degeneration, which implies that this high Efr3a expression may be important to the onset of spiral ganglion degeneration after hair cell loss [Nie et al., 2015].

\section{Conclusion}

For the first time, an association analysis with correction for population structure was used to map several loci for hearing traits in inbred strains of mice based on DPOAE suprathreshold amplitudes before and after noise exposure. Our results identified a number of novel loci for susceptibility to NIHL. Also, our findings validate the power of the HMDP for detecting NIHL susceptibility candidate genes. 


\section{Statement of Ethics}

This study was carried out in strict accordance with the recommendations of the American Association for Laboratory Animal Sciences. The protocol was approved by the University of Southern California Institutional Animal Care and Use Committee (Permit Number: 12033) and the Department of Animal Resources.

Animals were housed with ambient noise not exceeding that of normal air conditioning. All techniques were performed on mice under intraperitoneal anesthesia (ketamine $80 \mathrm{mg} / \mathrm{kg}$ body weight and xylazine $16 \mathrm{mg} / \mathrm{kg}$ body weight), and all efforts were made to minimize suffering.

\section{Conflict of Interest Statement}

The authors have no conflicts of interest to declare.

\section{Funding Sources}

This work was supported by National Institutes of Health grant NIDCD R01DC010856-01 to R.A.F. The funders had no role in study design, data collection and analysis, decision to publish, or preparation of the manuscript.

\section{Author Contributions}

Conceived and designed the experiments: J.L., H.A., and R.A.F. Performed the experiments: J.L., J.W., and R.A.F. Analyzed the data: J.L., G.K., R.F.B, A.M., J.W., A.L.C., H.A., and R.A.F. Contributed reagents/materials/analysis tools: J.L., G.K., A.M., R.F.B., J.W., A.L.C., and R.A.F. Wrote the paper: J.L., G.K., R.F.B., A.M., and R.A.F.

\section{References}

Ahmed M, Wong EY, Sol J, Xu J, Wang F, Xu PX. Eya1-Six1 interaction is sufficient to induce hair cell fate in the cochlea by activating Atoh1 expression in cooperation with Sox2. Dev Cell. 2012;22(2):377-90.

Bennett BJ, Farber CR, Orozco L, Kang HM, Ghazalpour A, Siemers N, et al. A high-resolution association mapping panel for the dissection of complex traits in mice. Genome Res. 2010;20:281-90.

Bennett BJ, Wang SS, Wang X, Wu X, Lusis AJ. Genetic regulation of atherosclerotic plaque size and morphology in the innominate artery of hyperlipidemic mice. Arterioscler Thromb Vasc Biol. 2009 Mar;29(3):348-55.

Davis RC, van Nas A, Bennett B, Orozco L, Pan C $\mathrm{Rau} C D$, et al. Genome-wide association mapping of blood cell traits in mice. Mamm Genome. 2013 Apr;24(3-4):105-18.

Erway LC, Shiau YW, Davis RR, Krieg EF. Genetics of age-related hearing loss in mice. III. Susceptibility of inbred and F1 hybrid strains to noise-induced hearing loss. Hear Res. 1996; 93:181-7.

Farber CR, Bennett BJ, Orozco L, Zou W, Lira A, Kostem E, et al. Mouse genome-wide association and systems genetics identify Asxl2 as a regulator of bone mineral density and osteoclastogenesis. PLoS Genet. 2011;7(4): e1002038.

Heinonen-Guzejev M, Vuorinen HS, MussaloRauhamaa H, Heikkilä K, Koskenvuo M, Kaprio J. Somatic and psychological characteristics of noise-sensitive adults in Finland Arch Environ Health. 2004;59(8):410-7.

Holme RH, Steel KP. Progressive hearing loss and increased susceptibility to noise-induced hearing loss in mice carrying a Cdh23 but not a Myo7a mutation. J Assoc Res Otolaryngol. 2004;5(1):66-79.

Hu H, Ma Y, Ye B, Wang Q, Yang T, Lv J, et al. The role of Efr3a in age-related hearing loss. Neuroscience. 2016. Nov 17;341:1-8.
Hu H, Ye B, Zhang L, Wang Q, Liu Z, Ji S, et al. Efr3a insufficiency attenuates the degeneration of spiral ganglion neurons after hair cell loss. Front Mol Neurosci. 2017;10:86.

Kang HM, Zaitlen NA, Wade CM, Kirby A, Heckerman D, et al. Efficient control of population structure in model organism association mapping. Genetics. 2008;178:1709-23.

Kochhar A, Fischer SM, Kimberling WJ, Smith RJ. Branchio-oto-renal syndrome. Am J Med Genet A. 2007;143A:1671-8.

Kozel PJ, Davis RR, Krieg EF, Shull GE, Erway LC. Deficiency in plasma membrane calcium ATPase isoform 2 increases susceptibility to noise-induced hearing loss in mice. Hear Res. 2002;164:231-9.

Kujawa SG, Liberman MC. Adding insult to injury: cochlear nerve degeneration after "temporary" noise-induced hearing loss. J Neurosci. 2009;29(45):14077-85.

Lavinsky J, Crow AL, Pan C, Wang J, Aaron KA, Ho MK, et al. Genome-wide association study identifies nox 3 as a critical gene for susceptibility to noise-induced hearing loss. PLoS Genet. 2015;11(4):e1005094.

Liberman MC. Noise-induced and age-related hearing loss: new perspectives and potential therapies. F1000Res. 2017;6:927.

Nie C, Hu H, Shen C, Ye B, Wu H, Xiang M. Expression of EFR3A in the mouse cochlea during degeneration of spiral ganglion following hair cell loss. PLoS One. 2015;10(1):e0117345.

Ohlemiller KK, McFadden SL, Ding DL, Flood DG, Reaume AG, Hoffman EK, et al. Targeted deletion of the cytosolic $\mathrm{Cu} / \mathrm{Zn}$-superoxide dismutase gene (Sod1) increases susceptibility to noise-induced hearing loss. Audiol Neurootol. 1999;4(5):237-46.

Ohlemiller KK, McFadden SL, Ding DL, Lear PM, Ho YS. Targeted mutation of the gene for cellular glutathione peroxidase (Gpx1) increases noise-induced hearing loss in mice. I Assoc Res Otolaryngol. 2000;1(3):243-54.
Park CC, Gale GD, de Jong S, Ghazalpour A, Bennett BJ, Farber CR, et al. Gene networks associated with conditional fear in mice identified using a systems genetics approach. BMC Syst Biol. 2011;5:43.

Parks BW, Nam E, Org E, Kostem E, Norheim F, Hui ST, et al. Genetic control of obesity and gut microbiota composition in response to high-fat, high-sucrose diet in mice. Cell Metab. 2013;17(1):141-52.

Rau CD, Parks B, Wang Y, Eskin E, Simecek P, Churchill GA, et al. High-density genotypes of inbred mouse strains: improved power and precision of association mapping. G3 (Bethesda). 2015;5(10):2021-6.

Sliwinska-Kowalska M, Pawelczyk M. Contribution of genetic factors to noise-induced hearing loss: a human studies review. Mutat Res. 2013;752:61-5.

White $\mathrm{CH}$, Ohmen JD, Sheth S, Zebboudj AF, McHugh RK, Hoffman LF, et al. Genomewide screening for genetic loci associated with noise-induced hearing loss. Mamm Genome. 2009;20(4):207-13.

Xu J, Ueno H, Xu CY, Chen B, Weissman IL, Xu PX. Identification of mouse cochlear progenitors that develop hair and supporting cells in the organ of Corti. Nat Commun. 2017;8: 15046.

Yankaskas K. Prelude: noise-induced tinnitus and hearing loss in the military. Hear Res. 2013Jan; 295:3.

Zhang Y, Knosp BM, Maconochie M, Friedman RA, Smith RJ. A comparative study of Eyal and Eya4 protein function and its implication in branchio-oto-renal syndrome and DFNA10. J Assoc Res Otolaryngol. 2004;5(3): 295-304.

Zheng W, Huang L, Wei ZB, Silvius D, Tang B, $\mathrm{Xu}$ PX. The role of Six 1 in mammalian auditory system development. Development. 2003;130(17):3989-4000. 